\title{
Leibniz rule, locality and supersymmetry on lattice
}

\section{Mitsuhiro Kato}

Institute of Physics, University of Tokyo, Komaba, Meguro-ku, Tokyo 153-8902, Japan

E-mail: kato@hep1.c.u-tokyo.ac.jp

\section{Makoto Sakamoto}

Department of Physics, Kobe University, Nada-ku, Hyogo 657-8501, Japan

E-mail: dragon@kobe-u.ac.jp

\section{Hiroto So*i}

Department of Physics, Ehime University, Bunkyou-chou 2-5, Matsuyama 790-8577, Japan

E-mail: so@phys.sci.ehime-u.ac.jp

In a finite volume system, we prove a no-go theorem on a Leibniz rule with a care of locality argument on latttice. The new possibility on the Leibniz rule solutions on lattice is discussed. Although the new solution admits a local difference operator, a non-local product rule is needed. In the case, a supersymmetric interacting theory is simply realized. The difference between finite flavor systems and matrix representations of infinite flavor systems is explained based on a finite volume system analysis including the no-go theorem.

The XXX International Symposium on Lattice Filed Theory

June 24-29,2012

Cairns, Australia

\footnotetext{
* Speaker.

$\dagger$ This work was supported in part by Grants-in-Aid for Scientific Research ( No.20340048, No.20540274 and No.22540281) by the Japanese Ministry of Education, Science, Sports and Culture.
} 


\section{Motivations}

When we construct interacting supersymmetric theories on lattice, we must take care of a Leibniz rule on lattice. A no-go theorem on the rule for an infinite volume system on lattice has been proved by us [1]. In its proof, there are two important clues, i.e. translational invariance and locality for an infinite system. The holomorphic function property associated with a lattice operator can decsribe those both clues. To manipulate the locality in a finite volume system, we must seek another discrete version of holomorphism which expresses translational invariance and locality. Corresponding to a certain translational invariant operator, a discrete function instead of a complex function can be defined and we can describe it as a local operator in the finite volume system.

There is a puzzling situation between a multi-flavor system and the matrix representation of an infinite flavor system which matrix product and a commutator difference operator is satisfied with the Leibniz rule [2]. To the contrary, there is a Leibniz rule no-go theorem on lattice for the finite flavor case. Cannot we recognize a matrix representaion as inifinite flavor number limit of a multi-flavor system? In order to solve the problem, we must analyze the finite number case of the flavor $N_{f}$ and the lattice size $N$ which implies the spatial volume.

\section{Definition of a local lattice theory}

In this article, we treat a one-dimensional system. The extention to higher dimensions can be realized by the direct product of higher dimensional coordinates. We shall start with a setup for a local lattice theory in a finite system. The system size and lattice constant are denoted as $N$ and $a_{L}=1$, respectively. We impose a the periodic boundary condition, $\phi_{n}=\phi_{n+N}$ on any lattice field.

A general difference operator on lattice is defined as

$$
(D \phi)_{n} \equiv \sum_{m}^{N} D_{n m} \phi_{m}
$$

where $\sum_{m}^{N} D_{n m}=0$ due to a vanishing constant mode. For a product rule, we generally define as

$$
(\phi \times \eta)_{n} \equiv \sum_{m \ell}^{N} C_{n m \ell} \phi_{m} \eta_{\ell}
$$

In the next step, we concentrate on translational invariant theories from general lattice ones ${ }^{1}$. As the result, the difference operator and the product rule have the following property,

$$
D_{n m}=D_{n+k m+k}=D(n-m)=D(n-m+N)
$$

and

$$
C_{n m \ell}=C_{n+k m+k \ell+k}=C(n-\ell, m-\ell)=C(n-\ell+N, m-\ell)=C(n-\ell, m-\ell+N),
$$

\footnotetext{
${ }^{1}$ The translational invariance strongly connects with the momentum conservation law. The strange momentum conservation on lattice can be realized as 134 .
} 
where the periodic boundary condition is used. By $N$-root of unity,

$$
w_{a} \equiv e^{\frac{2 \pi i a}{N}}=\omega_{N}^{a}, z_{b} \equiv e^{\frac{2 \pi i b}{N}}=\omega_{N}^{b}, \omega_{N} \equiv e^{\frac{2 \pi i}{N}}, \omega_{N}^{N}=1,
$$

we define "momentum" representations and their complex extentions,

$$
\begin{aligned}
\hat{D}_{a} & \equiv \sum_{m}^{N} w_{a}^{m} D(m)=\sum_{m}^{N} \omega_{N}^{a m} D(m), \\
\hat{D}_{a+i \varepsilon N} & \equiv \sum_{m}^{N} \omega_{N}^{(a+i \varepsilon N) m} D(m), \\
\hat{C}_{a b} & \equiv \sum_{m, n}^{N} w_{a}^{m} z_{b}^{n} C(n, m)=\sum_{m, n}^{N} \omega_{N}^{a m+b n} C(n, m), \\
\hat{C}_{a+i \varepsilon N, b+i \eta N} & \equiv \sum_{m, n}^{N} \omega_{N}^{(a+i \varepsilon N) m+(b+i \eta N) n} C(n, m),
\end{aligned}
$$

of the difference operator and the product rule where we must note that indices $a, b$ are discrete momentum labels. The locality in a finite volume system is defined as

$$
|D(-n)| \leq K e^{-\kappa|n|}, \kappa>0, \text { for } 1<<|n|<<N,
$$

for large $N$.

Using the orthogonality and the completeness property,

$$
\sum_{a}^{N} \omega_{N}^{(n+m) a}=N \delta_{n+m, 0}, \quad \sum_{n}^{N} \omega_{N}^{(a+b) n}=N \delta_{a+b, 0},
$$

the sufficient condition for locality in the finite system is that there exists nonzero finite $\varepsilon$ with

$$
\max _{a}\left\{\left|\hat{D}_{a+i \varepsilon N}\right|\right\}=O\left(N^{0}\right), \max _{a, b}\left\{\left|\hat{C}_{a+i \varepsilon N, b+i \eta N}\right|\right\}=O\left(N^{0}\right),
$$

because

$$
\begin{array}{r}
\frac{1}{N} \sum_{a}^{N} \omega_{N}^{n(a+i \varepsilon N)} \hat{D}_{a+i \varepsilon N}=\frac{1}{N} \sum_{a}^{N} \omega_{N}^{a n} \hat{D}_{a}=D(-n), \\
|D(-n)| \leq \frac{1}{N} \sum_{a}^{N}\left|\hat{D}_{a+i \varepsilon N}\right| e^{-2 \pi \varepsilon|n|} \leq \max _{a}\left\{\left|\hat{D}_{a+i \varepsilon N}\right|\right\} e^{-2 \pi \varepsilon|n|},
\end{array}
$$

where the first equality is similar to the Cauchy's integral theorem about a complex function owing to independence on $\varepsilon$. For the rule $C$, there are similar inequalities. On the other hand, in the case of

$$
|D(-n)| \leq K e^{-\kappa|n|}, \kappa>0, \text { for } 1<<|n|<<N,
$$

with

$$
\kappa \pm 2 \pi \varepsilon>0
$$


its necessary condition can be verified as

$$
\left|\hat{D}_{a+i \varepsilon N}\right|=\left|\sum_{m}^{N} \omega_{N}^{m(a+i \varepsilon N)} D(m)\right| \leq \sum_{m}^{N} e^{-2 \pi \varepsilon m}|D(m)| \leq K \sum_{m}^{N} e^{-(\kappa \pm 2 \pi \varepsilon)|m|}=O\left(N^{0}\right) .
$$

For product rule, similarily, in the case of

$$
|C(-m,-n)| \leq K e^{-\kappa|m|-\lambda|n|}, \quad \kappa>0, \lambda>0,
$$

with

$$
\kappa \pm 2 \pi \varepsilon>0, \lambda \pm 2 \pi \eta>0,
$$

the necessary condition can be verified as

$$
\begin{aligned}
\left|\hat{C}_{a+i \varepsilon N, b+i \eta N}\right| & =\left|\sum_{m, n}^{N} \omega_{N}^{m(a+i \varepsilon N)+n(b+i \eta N)} C(m, n)\right| \\
& \leq \sum_{m, n}^{N} e^{-2 \pi(\varepsilon m+\eta n)}|C(m, n)| \\
& \leq K \sum_{m, n}^{N} e^{-(\kappa \pm 2 \pi \varepsilon)|m|-(\lambda \pm 2 \pi \eta)|n|}=O\left(N^{0}\right) .
\end{aligned}
$$

In the summary of this section, we have proposed that (2.9) is the necessary and sufficient conditions for the locality in a finite volume system.

\section{Finite size no-go theorem for Leibniz rule on lattice}

A no-go theorem states that translation invariance, locality, a Leibniz rule and nontrivial product cannot be simultaneously satisfied on a finite volume lattice. The Leibniz rule by using only translation invariance condition can be rewritten as

$$
\hat{C}_{a, b}\left(\hat{D}_{a+b}-\hat{D}_{a}-\hat{D}_{b}\right)=0 .
$$

For any $a$ and $b$, if $\hat{C}_{a, b} \neq 0$, then (3.1) says

$$
\hat{D}_{a+b}-\hat{D}_{a}-\hat{D}_{b}=0 .
$$

The general solution of 3.2)is given by

$$
\hat{D}_{a}=\frac{a}{b} \hat{D}_{b} \propto a .
$$

The solution (3.3) is SLAC-type [5] owing to

$$
\omega_{N}^{a}=e^{\frac{2 \pi i a}{N}}=e^{i p} \Rightarrow a \propto p,
$$

and is non-local because of

$$
\max _{a}\left\{\left|\hat{D}_{a+i \varepsilon N}\right|\right\} \geq \frac{1}{N} \sum_{a}^{N}\left|\hat{D}_{a+i \varepsilon N}\right|=\frac{1}{N} \sum_{a}^{N}|a+i \varepsilon N|\left|\frac{\hat{D}_{b}}{b}\right|=O\left(N^{1}\right) \neq O\left(N^{0}\right) .
$$


If $\hat{C}_{a, b}$ is nontrivial, then $D(n)$ is SLAC-type and it is nonlocal. q.e.d

One of possible solutions may be the case that $D$ is local but $C$ is trivial or nonlocal. We can illustrate one example: $\hat{C}_{a, b}=K \delta_{a+b, 1}$. If $K=O\left(N^{0}\right)$, then the product rule is trivial which leads us to a trivial continuum limit. On the other hand, if $K=O\left(N^{1}\right)$, then it is just nonlocal. The real space expression is

$$
C_{l m n}=C(l-m, l-n)=\frac{K}{N} \delta_{m, n} \omega_{N}^{-(l-n)} .
$$

Its Leibniz rue realization is the followings,

$$
\hat{D}_{1}=\hat{D}_{a}+\hat{D}_{1-a} .
$$

This relation is not difficult to construct its local solutions. Using one of these solutions, we can write an explicit supersymmetric interacting action,

$$
\begin{array}{r}
S=\frac{1}{2} D \phi \cdot D \phi+i \bar{\psi} \cdot D \psi+F \cdot F+\frac{i g}{2} F \cdot(\phi \times \phi)+i g \phi \cdot(\bar{\psi} \times \psi) \\
\phi \cdot \chi \equiv \sum_{n}^{N} \phi_{n} \chi_{n},(\phi \times \chi)_{n} \equiv \sum_{m, l}^{N} C_{n m l} \phi_{m} \chi_{l}
\end{array}
$$

where the supersymmetry can be defined as

$$
\begin{aligned}
\delta \phi & =\varepsilon \bar{\psi}+\psi \bar{\varepsilon} \\
\delta \psi & =\varepsilon(i D \phi+F), \delta \bar{\psi}=\bar{\varepsilon}(-i D \phi+F), \\
\delta F & =-i \varepsilon D \bar{\psi}-i \bar{\varepsilon} D \psi .
\end{aligned}
$$

\section{Multi-flavor system and matrix representation}

In a finite multi-flavor system on lattice, no-go theorem on a Leibniz rule can be proved [1] but there exists the Lebniz rule through a matrix product rule in the matrix representation of an infinite flavor system [2]. This apparent inconsistency or the curious flavor limit can be solved by classifying two kinds of flavors after appropriate flavor diagonalization.

For a finite flavor $N_{f}$ and a finite volume $N$ system, product rule and a difference operator are defined as

$$
(\varphi \times \chi)_{l}^{p} \equiv \sum_{m, n, q, r}^{N, N_{f}} C_{l m n}^{p q r} \varphi_{m}^{q} \chi_{n}^{r},(D \varphi)_{l}^{p} \equiv \sum_{m, q}^{N, N_{f}} D_{l m}^{p q}
$$

where indices $p, q, r$ are flavor ones. From these translation invariance, we introduce the following notation,

$$
C_{l m n}^{p q r}=C^{p q r}(m-l, n-l), D_{l m}^{p q}=D^{p q}(m-l) .
$$

The $w$-representations $(N$-root expressions) of the product rule and the difference operator are expressed as

$$
\hat{C}_{L M}^{p q r} \equiv \sum_{m, n}^{N} \omega_{N}^{L m+M n} C^{p q r}(m, n), \hat{D}_{L}^{p q} \equiv \sum_{m}^{N} \omega_{N}^{L m} D^{p q}(m) .
$$


Their flavor matrix forms are

$$
\left(\hat{C}_{L M}^{q}\right)_{p r} \equiv \hat{C}_{L M}^{p q r},\left(\hat{D}_{L}\right)_{p q} \equiv \hat{D}_{L}^{p q}
$$

The Jordan's standard form of $\hat{D}$ leads us to the following parametrization in the flavor matrix,

$$
\hat{D} \rightarrow U \hat{D} U^{-1}=\hat{D}_{d i a g}+\hat{E}_{+}
$$

where

$$
\hat{D}_{\text {diag }}^{p q}=\hat{\Delta}_{L}^{p} \delta_{p q}, \quad \hat{E}_{+}^{p q}=\varepsilon_{L}^{p} \delta_{p, q+1}, \quad \hat{\Delta}_{0}^{p}=0, \varepsilon_{L}^{p}=0 \text { or } 1 .
$$

Then, we can write the Leibniz rule by a flavor matrix form

$$
\left(\hat{\Delta}_{L+M}^{p}-\hat{\Delta}_{L}^{q}-\hat{\Delta}_{M}^{r}\right) \hat{C}_{L, M}^{p q r}=\hat{R}_{L, M}^{p q r}(\varepsilon)
$$

where

$$
\hat{R}_{L, M}^{p q r}(\varepsilon) \equiv-\varepsilon_{L+M}^{p} \hat{C}_{L, M}^{p-1}{ }^{q r}+\varepsilon_{L}^{q+1} \hat{C}_{L, M}^{p q+1 r}+\varepsilon_{M}^{r+1} \hat{C}_{L, M}^{p q r+1}
$$

For a finite flavor system, since

$$
1 \leq p, q, r \leq N_{f}, \hat{C}_{L, M}^{0 q r}=\hat{C}_{L, M}^{p N_{f}+1 r}=\hat{C}_{L, M}^{p q N_{f}+1}=0
$$

it follows as

$$
R_{L, M}^{p q r}(\varepsilon)=0 .
$$

Therefore, the Leibniz rule of a finite flavor by a flavor matrix form is rewritten as

$$
\left(\hat{\Delta}_{L+M}^{p}-\hat{\Delta}_{L}^{q}-\hat{\Delta}_{M}^{r}\right) \hat{C}_{L, M}^{p q r} \equiv \hat{D}_{L, M}^{p q r} \hat{C}_{L, M}^{p q r}=0 .
$$

The solution is easily found as

$$
\hat{D}_{L, M}^{p q r}=0 \text { or } \hat{C}_{L, M}^{p q r}=0 .
$$

$\hat{D}_{L, M}^{p q r}=0$ case leads us to $\hat{\Delta}^{p}\left(w_{a}\right)=0$ in the local lattice theory framework, using a finite system no-go theorem which is proved in the previous section. Consequently, for a finite-flavored infinite volume system, we have the following two kinds of flavors; flavor type-A means that it has a trivial difference operator, $\hat{\Delta}^{p}(w)=0$ and flavor type-B does a trivial field product $\hat{C}_{L, M}^{p q r}=0$ between its flavors.

The next stage is the analysis for the matrix representation of an infinite flavor and infinite volume system. In the representation, we treat field variables, $\Phi_{i j}$ where both $i$ and $j$ run from 1 to $N_{\text {matrix }}$. We must consider an $N=N_{f}=2 N_{\text {matrix }}$ case because the following identification is realized,

$$
\Phi_{i j}=\phi_{n=(i+j)}^{p=(i-j)} .
$$

The product rule between matrices leads us to

$$
C_{l m n}^{p q r} \propto \delta_{p, q+r} \delta_{n-l, q} \delta_{l-m, r},
$$

and the commutator difference operator corresponds to 


$$
[d, \Phi]_{i j}=(D \phi)_{n=(i+j)}^{p=(i-j)},
$$

where $d$ implies some anti-hermitian $N_{\text {matrix }} \times N_{\text {matrix }}$ matrix. For this matrix representation, since we impose the usual periodic boundary condition for both a lattice space and a flavor space, the following relation

$$
R_{L, M}^{p q r}(\varepsilon) \neq 0
$$

is generated inevitably. This relation is the essential difference for usual finite flavor systems. Furthermore, owing to $R \neq 0$, there is always mixing between coupling-free flavor-B and motionfree flavor-A.

\section{Summaries}

We have proved a Leibniz rule no-go theorem in a finite volume system. Instead of holomorphic functions for infinite volume systems, we used the discrete bounded functions. Then, we can classify cases keeping the rule on lattice into the following three ones:

1 If we take a local nontrivial product $C_{l m n}$, then $D_{m n}$ is always SLAC-type (nonlocal).

2 If $D_{m n}$ is local, then $C_{l m n}$ is nonlocal or trivial. New possibility supersymmetry application with the strange momentum conservation law [3, 4].

3 If $D_{m n}$ is SLAC-type (nonlocal), then $C_{l m n}$ is arbitrary.

In the case of the second possibility, we can construct an explicit supersymmetric action with interactions.

For a finite flavor system versus the matrix representation in the infinite flavor system, we make a table:

\begin{tabular}{|c|c|c|c|c|}
\hline & $\begin{array}{c}\text { the number of } \\
\text { components }\end{array}$ & Leibniz rule & locality & A-B separation \\
\hline multi flavor & $N_{f} \times N$ & no-go & local & yes \\
\hline multi flavor & $N_{f} \times N$ & no-go & nonlocal & yes \\
\hline $\begin{array}{c}\text { matrix } \\
\text { representation }\end{array}$ & $\begin{array}{c}N \times N \\
=N_{\text {matrix }} \times N_{\text {matrix }}\end{array}$ & escape & $\begin{array}{c}\text { nonlocal } \\
\text { by } N \text { infinity }\end{array}$ & no \\
\hline
\end{tabular}

\section{References}

[1] M. Kato, M. Sakamoto, and H. So, Taming the Leibniz Rule on the Lattice, JHEP 0805(2008) 057.

[2] M. Kato, M. Sakamoto and H. So, Leibniz rule and exact supersymmetry on lattice: A Case of supersymmetrical quantum mechanics, PoS LAT2005 (2006) 274.

[3] P.H. Dondi and H. Nicolai, Lattice Supersymmetry, Nuovo Cim. A41 (1977) 1.

[4] A. D’Adda, I. Kanamori, N. Kawamoto and J. Saito Species Doublers as Super Multiplets in Lattice Supersymmetry: Chiral Conditions of Wess-Zumino Model for D=N=2, JHEP 0805(2008) 057.

[5] S.D. Drell, M. Weinstein and S. Yankielowicz, Strong-coupling field theories. II. Fermions and gauge fields on a lattice, Phys. Rev. D14 (1976) 1627. 